\title{
Vorbemerkung der Herausgeber
}

Der vorliegende Band enthält ausgewählte Beiträge des ersten Symposiums zum Thema „Das Buch des Dede Korkut und das Nibelungenlied - ein interkultureller Epenvergleich", das vom 30. September bis zum 4. Oktober 2009 in Baku von der dortigen Slawistischen Universität veranstaltet wurde. Dieses erste Symposium war sprachwissenschaftlichen Aspekten der beiden Epen gewidmet. Als Fortsetzung sind Symposien zu literaturwissenschaftlichen Aspekten und zur Rezeptionsgeschichte in Vorbereitung. Die Veranstaltungsreihe wird getragen von einer projektmäßigen Zusammenarbeit zwischen der Slawistischen Universität Baku, dem Deutschen Institut und dem Seminar für Orientkunde der Johannes Gutenberg-Universität Mainz sowie der Oswald von Wolkenstein-Gesellschaft, Frankfurt am Main. Die Durchführung des interkulturellen Symposiums ist durch eine großzügige Beihilfe von der Deutschen Forschungsgemeinschaft ermöglicht worden. Für die Förderung des Projekts waren mehrere Gründe ausschlaggebend: Erstens handelt es sich bei diesem Vorhaben um das erste bilaterale Wissenschaftsprojekt zwischen Deutschland und Aserbaidschan. Zudem ist damit ein Projekt auf den Weg gebracht, das die Gräben zwischen den Wissenschaftsdiskursen in West und Ost zu überbrücken trachtet.

Die einzelnen Beiträge beschäftigen sich mit Themen, die überwiegend auf sprachwissenschaftlichen Analyseebenen angesiedelt sind, wobei die Sprachwissenschaft hier im weitesten Sinne aufgefasst wurde: syntaktische Strukturen und lexikographische Gesichtspunkte kommen genauso zum Tragen wie Sprechakttheorie, der sprachliche Hintergrund der poetischen Strukturen oder die Onomastik der Heldennamen.

Daneben enthält der Band Beiträge, die darauf angelegt sind, den am Symposium teilnehmenden Germanisten einerseits und den Turkologen und aserbaidschanischen Sprachwissenschaftlern anderseits den Hintergrund der jeweiligen Forschungsgegenstände zu vermitteln. Dabei werden Einzelaspekte wie die Metrik der Nibelungenstrophe aus germanistisch-deutscher wie aus germanistisch-aserbaidschanischer Perspektive beleuchtet. Außerdem werden übergreifende Themen wie die Wichtigkeit des Nibelungenliedes für die internationale Germanistik angesprochen sowie der Stellenwert des Buches des Dede Korkut als Verschriftlichung einer mündlichen Überlieferung für die aserbaidschanische Literaturgeschichte und für das Studium des Aserbaidschanischen als Zweig des Westogusisch-Türkischen erörtert.

Begleitend zum Symposium fand am 2. Oktober 2009 ein Konzert im Kapellhaus, dem deutschen Kulturzentrum in Baku, statt, bei dem der bekannte Nibelungeninterpret Eberhard Kummer und die aserbaidschanische Musikgruppe Savalan in einem vor Ort improvisierten musikalischen Austausch ihre Interpretationen der epischen Erzählstoffe darboten. Am Ende des Bandes findet sich ein Beitrag, in dem Eberhard Kummer und Johannes Rettelbach von diesem Ereignis berichten.

Kamal M. Abdullayev, Baku

Hendrik Boeschoten und Uta Störmer-Caysa, Mainz Sieglinde Hartmann, Frankfurt am Main 